\title{
'We were able to gain a greater appreciation for emergency medicine'
}

\section{Molly Watton, DCT in Oral and Maxillofacial Surgery and General Duties, The Royal London Hospital, London, UK, describes her second round of redeployment, in the ICU.}

\footnotetext{
S
} taff at The Royal London Dental Hospital were redeployed to support critical care and the wards during both the first and second waves of the pandemic. The second wave saw a peak which was higher and lasted for longer than the first, necessitating renewed vigour, energy and motivation of redeployed staff towards the national fight against COVID-19. It was agreed with the London Ambulance Service that The Royal London Hospital (RLH) would be the main receiving centre for the most serious COVID-19 patients, as well as trauma and emergency patients within Barts Health NHS Trust. ${ }^{1}$ The Royal London Hospital tripled its total critical care capacity from a baseline of 44 beds to around 150 beds at the peak, utilising the COVID-19 critical care surge facility, the Queen Elizabeth Unit.

All members of the dental team were redeployed to provide support where required. Restorative and oral and maxillofacial surgery dental core trainees (DCTs) were redeployed to healthcare support worker roles and intensive care doctor support roles, respectively. This involved 12.5-hour shifts, both day and night in COVID-19 critical care units and the adult intensive care unit across a three-month period from January to March 2021. It was an anxiety provoking time in our training, where DCTs were out of their comfort zone and had to learn new skills to keep up with increasing demand.

Healthcare assistant roles ranged from proning patients, patient hygiene and cleaning, taking observations, liaising with family members and other general ward duties. Junior doctor duties involved treatment planning with other members of the critical care team, liaising with multidisciplinary teams including microbiology, radiology and theatre teams, requesting special investigations - most commonly chest x-rays - hands-on tasks such as assisting with the placement of central and arterial lines, prescribing, liaising with family members and involvement in medical emergencies. While part of the critical care team, we also observed multiple intubations and extubations, tracheostomies, insertions of surgical chest drains and a one-off emergency caesarean section of an intubated patient with deteriorating COVID-19 pneumonia.

Our roles further evolved to include mouth care for patients, particularly important for those intubated. Dental teams used their knowledge and skills to care for the mouths and dentitions of patients who were critically unwell with COVID-19. COVID-19 complications are caused by a severe inflammatory reaction that share some common signals with periodontitis and evidence has shown that periodontitis is significantly associated with higher risk of complications from COVID-19, specifically ICU admission, need for assisted ventilation,

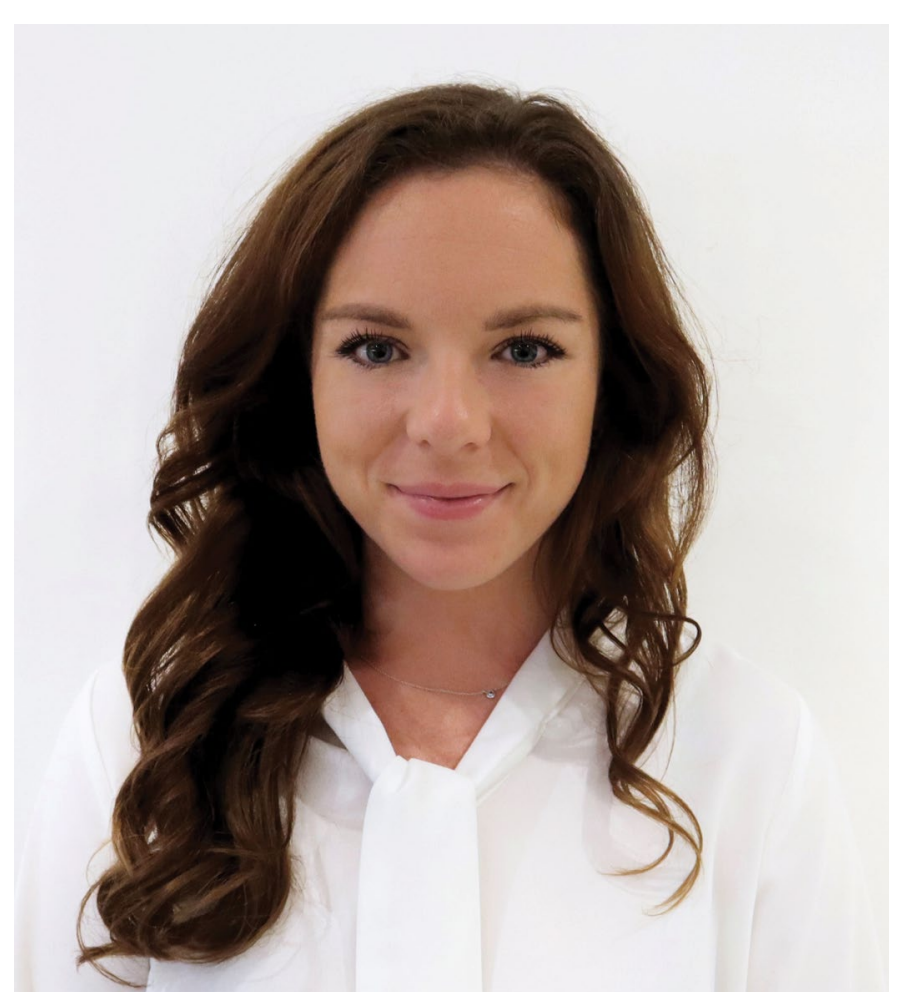

increased blood levels of biomarkers linked to worsening disease outcomes (D-dimer, white blood cell count and C-reactive protein levels) and death. ${ }^{2}$ The link between oral health and the severity of COVID-19, and the potential vascular route of transfer of SARSCoV-2 from the oral cavity to the lungs has further been hypothesised, whereby there is potential for translocation of the virus from the saliva reservoir to the gingival sulcus. This is enhanced by periodontal pockets and subgingival plaque biofilm, present in periodontitis, facilitating the survival of the virus and invasion of the oral mucosa immune response, resulting in the direct vascular entry of the virus into the pulmonary vessels which corresponds with the radiological characteristics of primary pulmonary vasculopathy.

To act against the viral reservoir in the mouth, oral hygiene and oral care is of utmost importance. The aim of mouth care for hospitalised patients is to maintain oral cleanliness, prevent the oral cavity from becoming dry and sore, prevent additional oral infections and importantly reduce the risk of developing COVID-19 associated bacterial pneumonia. ${ }^{4}$ Mouth care was given to both ventilated and non-ventilated patients, the difference being how independent the patient could be with their oral hygiene regime. Care for the intubated patient involved first checking the cuff on the endotracheal tube was inflated to prevent aspiration, brushing the teeth and using chlorhexidine mouthwash on sponges to access all areas of the mouth. Suction during the process, particularly at the back of the throat was vital. Lubricant was also applied to patients' eyes and lips 
$\$$ at regular intervals to prevent dryness. Non-intubated patients had a similar routine, however if the patient was able, they were also provided with a soft, small headed manual toothbrush to brush their teeth independently. Examination of the oral cavity and dentition was completed to ensure prostheses such as dentures had been removed prior to intubation. Furthermore, patients requiring extractions for troublesome or mobile teeth in close proximity to the endotracheal tube which posed an inhalation risk, were carried out on the ward too.

Many learning points can be taken from this redeployment opportunity, most notably gaining a deeper understanding in the management of medically compromised and critically ill patients. Although as dentists we do not have comprehensive knowledge of all medical conditions or the body as a whole as per doctors, upon registration with the General Dental Council, dentists are required to be able to identify general and systemic disease in addition to having a good knowledge of dental, oral, craniofacial and general anatomy. ${ }^{5}$ This knowledge linked heavily to what we witnessed and understood in the ICU, an environment which we had not experienced before. End of life care is not something as a dentist you deal with on a regular basis, however, it is something we became accustomed to during this period. Our communication skills were tested, both with family members and other clinicians in the medical team, as well as the emotional impact our surroundings had on us and all frontline NHS workers. Importantly, we were able to gain a greater appreciation for emergency medicine, medical emergencies, the airway and the importance of a multidisciplinary team response in patient care, skills which will be transferred to our future careers as developing dentists.

On 11 May 2021, after almost 28 weeks, the COVID-19 critical care surge facility at RLH, the Queen Elizabeth Unit, was stood down. Redeployed staff have returned to their usual departments and elective services have been reinstated. DCTs and other dental staff were given the opportunity to contribute their specialist knowledge of the oral cavity, the dentition and the head and neck region during this unprecedented time, in addition to learning many new skills, allowing for interprofessional working and strengthening of the mutual respect between dental and medical fields. Putting the interests and care of patients first has always been the priority, regardless of specialty, which has been highlighted by the dedication of healthcare workers. Frontline NHS workers have excelled in supporting our NHS, and from my own experience in the ICU, I would like to take this opportunity to express my pride and appreciation for all those who stepped up during this difficult and testing time. Redeployment and the fight against COVID-19, albeit beyond challenging and emotional at times, is an experience I am so proud to have been a part of and is something I, like many, will never forget.

\section{References}

1. Barts Health NHS Trust. A winter like no other... How Barts Health responded to the second wave of Covid-19. March 2021. Available at: https://www.bartshealth.nhs.uk/download. $\mathrm{cfm} ? \mathrm{doc}=$ docm93jijm4n16833.pdf\&ver=30223 (accessed May 2021).

2. Marouf N, Cai W, Said K N et al. Association between periodontitis and severity of COVID19 infection: A case-control study. J Clin Periodontol 2021; 48: 483-491.

3. Lloyd-Jones G, Molayem S, Pontes C C, Chapple I. The COVID-19 pathway: A proposed oral-vascular-pulmonary route of SARS-CoV-2 infection and the importance of oral healthcare measures. J Oral Med and Dent Res 2021; 2: 1-25.

4. Public Health England. Mouthcare for hospitalised patients with confirmed or suspected COVID-19. 6 August 2020. Available at: https://www.gov.uk/government/publications/ covid-19-mouth-care-for-patients-with-a-confirmed-or-suspected-case/mouth-care-forhospitalised-patients-with-confirmed-or-suspected-covid-19 (accessed May 2021).

5. Sacoor S, Chana S, Fortune F. The dental team as part of the medical workforce during national and global crises. Br Dent J 2020; 229: 89-92. 